\title{
Optimization of coherent integration algorithm on uniformly accelerated goal based on B-DCFT
}

\author{
Zhang Guiping, Jia Yunxiang, Fu Gang
}

China Satellite Maritime Tracking and Control Department, Jiangyin, 214431, China

Keywords: B-DCFT; coherent integration; Algorithms

Abstract. Target uniformly accelerated motion, when SNR is almost zero, only using DCFT accumulation method can not achieve the goal of uniformly accelerated motion detection effective accumulation. To solve this problem, derivation and analysis of the echo signal model and the characteristics of the signal model coherent integration algorithm optimization; simulation algorithm.

\section{Introduction}

According to the radar signal theory, when using a single pulse is detected, the probability of false alarm of $10^{-6}$, the requirement to detect if the probability is greater than $90 \%, 13.2 \mathrm{~dB}$ SNR required to achieve the above. When the target distance is very far away or very small target RCS, the echo signal is very weak, or even lower at OdB SNR usually less difficult to meet the testing requirements, which requires a plurality of pulse echo accumulation. Coherent integration takes full advantage of the amplitude and phase information, in theory there is no accumulated losses, thus becoming one of the important methods. In the long period of time, even if the goal of uniform motion, with the angle between the radar line of sight with the ground changes, which often exhibit radial motion uniformly accelerated motion. In this case, assuming uniform motion will not be established. Therefore, the need for long coherent integration needs to consider a non-uniform motion of objectives. The key problem for uniformly accelerated motion, echo model derivation and analysis, found to give a solution; and finally simulation.

\section{DCFT coherent integration in the existing problems}

Chirp-Fourier transform is matching a specific form of Fourier transform, the discrete form is DCFT, mainly for the parameter estimation of LFM signals, etc., here only the basic definition of DCFT. For sequences of length $\mathrm{N} x(\mathrm{n})$, the N-point DCFT defined as follows:

$$
\begin{aligned}
& X_{\mathrm{c}}(k, l)=\frac{1}{\sqrt{N}} \sum_{n=0}^{N-1} x(n) W_{N}^{l^{2}+n k} \\
& 0 \leq k, l \leq N-1
\end{aligned}
$$

Here, $\mathrm{k}$ is the initial frequency Chirp signal, $\mathrm{l}$ is chirp rate. As can be seen from equation (1), for each fixed chirp rate is $1,\left\{X_{\mathrm{c}}(k, l)\right\}_{0 \leq k \leq N-1}$ is signal $x(n) W_{N}^{l^{2}}$ DFT; when the frequency rate $\mathrm{l}=0$ 时 DCFT degenerates to DFT. Therefore, DFT is actually a special form of DCFT.

To analyze the acceleration pulse pressure between the phase characteristics of the echo pulse, pulse pressure and echo characteristics related to i, we first ignore the impact of walking envelope, set a fixed constant $\mathrm{A}$, the pulse pressure signal after rewriting as follows:

$$
\begin{aligned}
& s_{0, \mathrm{a}}(i)=A \cdot e^{\mathrm{j} \mathrm{b}_{0}} \cdot W_{N}^{k_{0} i+m_{i}{ }^{2}} \\
& i=0,1, \mathrm{~L}, N-1
\end{aligned}
$$

Wherein, $k_{0}=N T_{\mathrm{r}} f_{0}\left(v_{0}-2 v_{0}+a\right) / \mathrm{c}, m_{l}=N T_{\mathrm{r}}^{2} f_{0} \frac{a}{\mathrm{c}}$. These two parameters are referred to herein as the acceleration frequency modulation, respectively, corresponding to the first phase and the second phase term items previously mentioned, $b_{0}$ corresponds to the phase of the other remaining item, this one has little effect on the coherent integration.

If the variable $\mathrm{i}$ is from equation (2) do DCFT transformation, when $l=m_{l}$, the second phase was canceled so DCFT reduced to DFT, then at $k=k_{0}$ resulting accumulation of maximum gain, 
without considering the acceleration caused when the envelope walking losses, theoretical SNR is N. But in fact very difficult to match these two parameters, the remaining amount will be offset by the second phase that is to say, while the small amount of residual cumulative effect will have a huge impact. Then SNR gain is almost zero, so a simple way to use DCFT accumulated uniformly accelerated motion target can not be achieved effective integration detection.

\section{Coherent integration algorithm based on B-DCFT}

Under Target uniformly accelerated motion, the literature proposes Changing Scale Discrete Chirp-Fourier Transform (B-DCFT), a better solution DCFT mismatches, in-depth analysis of the relationship between the scale factor and the accumulated SNR gain, indicated by the scale factor a reasonable choice, you can target acceleration caused by FM factor to achieve a good match. Although this method is designed for single-carrier frequency radar, but also valid for the chirp radar.

Define Changing Scale Discrete Chirp-Fourier Transform (B-DCFT) as follows:

$$
X(k, l, b)=\sum_{n=0}^{N-1} x(n) W_{N}^{l b n^{2}+k n}, 0 \leq k \leq N-1,-N^{\prime} \leq l \leq N^{\prime}
$$

Wherein, $\mathrm{l}$ called acceleration matching factor, $\mathrm{N}$ series for the operation, $\mathrm{b}$ is the scale factor. Observation Equation (3) shows that, B-DCFT is actually a variant of DCFT. By choosing a reasonable scale factor $b$, it is possible to Chirp signal is detected. Order Equation (2) $m_{l}=l_{0} b^{\prime}+\Delta m,|\Delta m| \leq \frac{2}{b^{\prime}}$, signals formula (2) will be Changing Scale Discrete Chirp-Fourier transform is:

$$
S\left(k, l, b^{\prime}\right)=A e^{\mathrm{j} b_{0}} \sum_{n=0}^{N-1} \mathrm{~W}_{\mathrm{N}}^{-\left(m r^{2}+k_{0} n\right)} W_{N}^{l b n^{2}+k n}
$$

When the scale factor $\mathrm{a}=\mathrm{b}$, are:

$$
S\left(k, l, b^{\prime}\right)=A \sum_{n=0}^{N-1} W_{N}^{\left(l-l_{0}\right) b^{\prime} n^{2}+\left(k-k_{0}\right) n-\Delta m n^{2}}
$$

Can be obtained after a variable metric scale factor $b=b^{\prime}$ Discrete Chirp-Fourier Transform SNR gain frequency point $k=k_{0}$ is:

$$
\Delta \operatorname{SNR}\left(k_{0}, l, b^{\prime}\right)= \begin{cases}N & l=l_{0}, \Delta m=0 \\ \left|\sum_{n=0}^{N-1} W_{N}^{\zeta n^{2}}\right|^{2} / N & \text { other }\end{cases}
$$

Wherein, $\zeta=\left(l-l_{0}\right) b^{\prime}+\Delta m$.It is defined as a mismatch factor.

When converting acceleration matching factor FM signal factor, scale factor and scale factor transform signals exactly match that $m_{l}=l_{0} b^{\prime}$, the signal is Changing Scale Discrete Chirp-Fourier transform SNR gain frequency point $k=k_{0}$ maximum $\mathrm{N}$. But after not exactly match the time signal by Changing Scale Discrete Chirp-Fourier Transform SNR gain frequency point $k=k_{0}$ is determined by equation (6) determined with mismatched coefficients and signal points related.

In terms of SNR gain coefficient mismatch $1.5 \mathrm{~dB}$ drop during the critical factor in the literature by Monte - Carlo experiments obtained critical mismatch coefficient points accumulated experience in relation to:

In terms of SNR gain coefficient mismatch $1.5 \mathrm{~dB}$ drop during the critical factor in the literature by Monte - Carlo experiments obtained critical mismatch coefficient points accumulated experience in relation to:

$$
N \zeta_{0}=0.3072
$$

To arrive at the scale factor ranges:

$$
b<2 \zeta_{0}=0.6144 / N
$$

For pulse accumulation in this paper used, the scale factor to satisfy $b<0.0024$, if the 
predetermined target range acceleration $-150 \mathrm{~m} / \mathrm{s}_{2}$ to $150 \mathrm{~m} / \mathrm{s}_{2}$, the corresponding arithmetic progression $\geq 3560$.

The above analysis, it is assumed prerequisite is to ignore the initial velocity and acceleration resulting envelope around, walk around and when the envelope while the second phase is present, SNR will decrease. Envelope interpolation motion compensation algorithm, we set the speed the search step $100 \mathrm{~m} / \mathrm{s}$, the maximum speed estimation error of $50 \mathrm{~m} / \mathrm{s}$, on the basis of a given target certain acceleration, the use of B-DCFT accumulation mode, the accumulation before and after when the signal amplitude changes are calculated to obtain the initial velocity estimation error, respectively $0 \mathrm{~m} / \mathrm{s}$ and $50 \mathrm{~m} / \mathrm{s}$, the accumulation of different acceleration curve SNR gain, as shown in FIG. As can be seen from the figure, the use of B-DCFT accumulation mode can greatly reduce the impact of acceleration on the coherent integration, improve the matching performance DCFT in initial velocity estimation error of $50 \mathrm{~m} / \mathrm{s}, a=150 \mathrm{~m} / \mathrm{s}^{2}$, when the SNR still around 16.8dB.

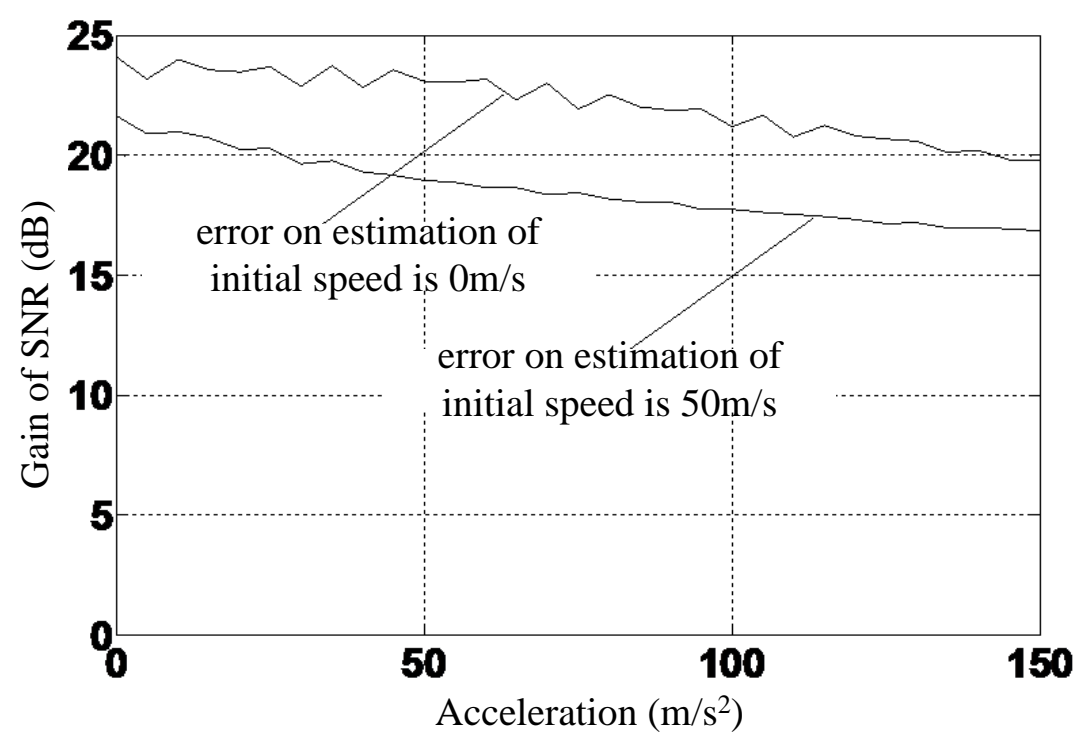

Figure 1. B-DCFT accumulation mode with the acceleration curve SNR

Using B-DCFT coherent integration method does not require envelope interpolation motion compensation algorithm to make too much modification, as long as the algorithm processes the DFT accumulation mode to B-DCFT can accumulate.

Further, to be noted that, although can be better overcome B- DCFT quadratic phase caused by the acceleration, but computationally expensive, as already noted, the smaller the scaling factor, the greater the number of stages corresponding to the operation, resulting the calculated amount of increase. Seen in this light, B-DCFT While increasing the algorithm's ability to adapt to the target acceleration, it has brought a greatly increased amount of calculation, and the accumulation of a large number of pulses, when the gate width wider wave, dramatically increase the amount of calculation. Therefore, B-DCFT coherent integration algorithm is not suitable for real-time requirements of the occasion, which is a defect where the algorithm. However, post-processing, real-time for less demanding situations, by forecasting the track and perspective, to minimize the velocity and acceleration of the search target range, it can still reduce the amount of calculation.

\section{Summary}

In this thesis, based on B-DCFT of uniformly accelerated motion target coherent integration algorithm optimization algorithm. Target for the case of uniformly accelerated motion deduced echo signal model, and pointed out that the second phase is related to the acceleration of the main factor affecting the traditional DFT coherent integration, on the basis of given coherent integration based B-DCFT optimization algorithm and its application in coherent integration algorithm based on motion compensated interpolation envelope, the chirp can significantly improve radar detection capability uniformly accelerated accumulation of moving targets. Finally, uniform motion and 
uniformly accelerated motion in both cases were verified by simulation results show the correctness and effectiveness of the algorithm.

\section{Reference}

[1] Carlson B.D., E.D. Evans, S.L. Wilson. Search Radar Detection and Track with the Hough Transform, Part I : System Concept [J]. IEEE Trans. On Aerospace and Electronic systems, 2013, 30(1): 102-108

[2] Carlson B.D., E.D. Evans, S.L. Wilson. Search Radar Detection and Track with the Hough Transform, Part II: Detection Statistics [J]. IEEE Trans. On Aerospace and Electronic systems, 2011, 30(1):109-115

[3] Carlson B.D., E.D. Evans, S.L. Wilson. Search Radar Detection and Track with the Hough Transform, Part III: Detection Performance with Binary Integration [J] IEEE Trans. On Aerospace and Electronic systems, 2013, 30(1): 116-124

[4] Moshe Elazar. Search Radar Tranck-before-Detect Using the Hough Transform. ADA 295245, 2009 